\title{
Mitochondrion- and nucleus-acting polymeric nanoagents for chemo-photothermal combination therapy
}

Xiaoyang Liu, Hao-Ran Jia, Ya-Xuan Zhu, Ge Gao, Yao-Wen Jiang, Xiaotong Cheng, Ke-Fei Xu, Xin-Wang $\mathrm{Yu}$ and $\mathrm{Fu}-\mathrm{Gen} \mathrm{Wu}^{*}$

\begin{abstract}
Developing intrinsically mitochondria-targetable nanosystems for subcellular structure-oriented precise cancer therapy is highly desirable. Here, we conjugate the cluster determinant 44 (CD44)-targetable hyaluronic acid (HA) with cholesterol-poly(ethylene glycol) ${ }_{2 \mathrm{k}}-\mathrm{NH}_{2}$ and mitochondria-acting IR825- $\mathrm{NH}_{2}$ (a cyanine dye) to construct a self-assembled nanostructure (abbreviated as HA-IR825Chol) for photothermal therapy. The HA-IR825-Chol exhibits improved photostability and desirable photothermal properties, and can rapidly and substantially enter CD44-overexpressed cancer cells and selectively accumulate in the mitochondria of the cells. Upon near-infrared laser irradiation, it can induce severe mitochondrial damage, which causes cytochrome $\mathrm{c}$ release and triggers cell apoptosis. Furthermore, we demonstrate the feasibility of loading the chemotherapeutics 10-hydroxycamptothecin (HCPT) into the hydrophobic cores of HA-IR825-Chol NPs for combined chemophotothermal therapy. HCPT encapsulated within HA-IR825Chol achieves significantly increased cellular uptake and simultaneous mitochondrial and nuclear localization, leading to the release of cytochrome $\mathrm{c}$ from mitochondria and upregulation of cleaved caspase-3, both of which contribute to the cell apoptosis/death. In vivo experiments reveal the excellent tumor-targeting ability of HA-IR825-Chol/HCPT, ensuring the efficient tumor eradication by the chemo-photothermal therapy. This work exemplifies the development of an intrinsically mitochondria-targetable nanocarrier for precise subcellular structure-localized drug delivery, and the Chol-mediated rapid and massive endocytosis of the nanoagents may represent a robust strategy for enhancing the efficacies of nanomedicines.
\end{abstract}

Keywords: combination cancer therapy, drug delivery, apoptotic pathway, cytochrome $c$, mitochondrial targeting

\section{INTRODUCTION}

Cancer as one of the most lethal diseases has severely threatened human health. As an essential organelle that participates in many cellular behaviors, mitochondria are indispensable for energy production in cells [1]. Notably, the number and metabolic activity of mitochondria in cancer cells have been unraveled to be higher than those in normal cells, and therefore targeting and destructing mitochondria in cancer cells represents an effective means for tumor therapy [2]. To date, a number of mitochondria-targeting nanomedicines or nanoparticles (NPs) have been developed [3-6]. Current ligands for the selective delivery of anticancer agents to the mitochondria of tumor cells mainly include lipophilic cations $[7,8]$, peptides $[9,10]$, aptamers [11], and intrinsically targetable nanoparticles [12]. In addition, among the different alternative strategies for anti-tumor treatments [13], photothermal therapy (PTT) plays an essential role as a noninvasive method for cancer therapy. The therapeutic efficiency of PTT usually depends on the photothermal agents (PTAs) that can convert light energy into thermal energy for cancer cell killing. Numerous PTAs have been developed, including metal-based PTAs [14-18], carbonbased PTAs [19-22], melanin-like NPs [23,24], conjugated polymers [25-27], black phosphorus [28,29], metal-organic frameworks [30], covalent organic frameworks [31], cyanine-based PTAs [32], and so on. However, some of these PTAs have shortcomings like tedious synthesis, non-degradable property, high cytotoxicity, and low photothermal conversion efficiency. Therefore, developing a suitable PTA that can address some or all of the above shortcomings is highly desirable. It has been reported that some cyanine-based molecules or composite

\footnotetext{
State Key Laboratory of Bioelectronics, School of Biological Science and Medical Engineering, Southeast University, Nanjing 210096, China * Corresponding author (email: wufg@seu.edu.cn)
} 
materials possess a strong near-infrared (NIR) absorption character and can realize not only phototherapy but also NIR fluorescence imaging under a single wavelength excitation. Cyanine-based agents mainly include heptamethine cyanine-based agents like indocyanine green [33-42], phthalocyanine-based agents [43-46], and others $[47,48]$. IR825, a heptamethine cyanine dye, shows high photothermal conversion efficiency, good biodegradability, mitochondria-targeting property, and fluorescence imaging capability, which are beneficial for cancer theranostics $[49,50]$. More importantly, mitochondria in cells are highly sensitive to hyperthermia [51], and mitochondria-targeted PTT can effectively influence the activity of enzymes, disturb mitochondrial respiration, induce mitochondrial thermal damage, and further activate mitochondrial apoptosis pathways [52,53]. Hence, mitochondria-targeted PTT represents a promising organelle-targeted phototherapy to fight against cancer. However, many previously developed mitochondrial targeting nanoagents involve the use of the cationic triphenylphosphonium (TPP) ligand. However, the TPP modification strategy has many practical problems such as unsatisfied repeatability, potential toxicity, and tedious chemical conjugation [49]. Therefore, developing a nanosystem with intrinsic mitochondrial targeting capability is highly desired.

On the other hand, hyaluronic acid (HA) is a linear, biodegradable, and biocompatible polysaccharide material, which is promising for drug delivery application [54]. $\mathrm{HA}$ is rich in carboxyl groups and it can recognize HA receptors (such as cluster determinant $44, \mathrm{CD} 44$ ) overexpressed in various types of tumor cells [55] and thus is widely used for targeting tumor tissues [56,57]. Till date, a variety of HA-based nanomaterials have been rationally designed for precise cancer therapy [58-62]. Therefore, using HA polymer to construct photothermal nanoagents holds great potential to realize tumor-selective PTT.

In this work, using the reaction between the carboxyl groups of $\mathrm{HA}$ and the amine-containing molecules, we conjugate HA with IR825- $\mathrm{NH}_{2}$ and cholesterol-poly (ethylene glycol) ${ }_{2 \mathrm{k}}-\mathrm{NH}_{2}\left(\right.$ Chol-PEG- $\mathrm{NH}_{2}$ ) to fabricate a self-assembled nanocarrier (termed HA-IR825-Chol) that specifically targets tumor cells and acts on mitochondria (Scheme 1). It should be pointed out that, Chol, a robust lipophilic anchor $[63,64]$, has been introduced to various nanosystems to interact with plasma membranes for enhanced cellular uptake [65-67]. Compared with IR825$\mathrm{NH}_{2}$, the as-designed HA-IR825-Chol NPs exhibited improved water-dispersity, photostability, and photothermal properties, and showed satisfying cancer cell targeting ability via the recognition between HA and the CD44 overexpressed in many tumor cells. Upon NIR laser irradiation, the IR825-mediated PTT could damage the mitochondrial structure, resulting in cytochrome $c$ release from mitochondria and tumor cell death. Furthermore, we demonstrated the feasibility of using HAIR825-Chol NPs to carry a chemotherapeutic drug 10hydroxycamptothecin (HCPT). It was found that the asformulated HA-IR825-Chol/HCPT NPs could realize IR825-mediated mitochondrial PTT and HCPT-mediated nucleus- and mitochondrion-oriented chemotherapy.

\section{EXPERIMENTAL SECTION}

\section{Synthesis of HA-IR825, HA-IR825-mPEG and HA-IR825- \\ Chol}

IR825- $\mathrm{NH}_{2}$ was synthesized according to a previous report [68]. To prepare HA-IR825, $20 \mathrm{mg}$ HA dissolved in $30 \mathrm{~mL}$ of deionized water was added with $94 \mathrm{mg} \mathrm{N}$-hydroxy-sulfosuccinimide sodium salt (sulfo-NHS) and $32 \mathrm{mg} N$-(3-dimethylaminopropyl)- $N$ '-ethylcarbodiimide hydrochloride (EDC. $\mathrm{HCl}$ ) to activate the carboxyl groups of HA. After $2 \mathrm{~h}, 5 \mathrm{mg}$ IR825- $\mathrm{NH}_{2}$ dissolved in a small amount of dimethyl sulfoxide (DMSO) was added into the above activated HA solution, and the obtained mixture was stirred at room temperature in the dark for $24 \mathrm{~h}$. After that, the mixture was dialyzed sequentially against "DMSO + deionized water" and deionized water by using a dialysis membrane (molecular weight cut-off (MWCO) $=3500)$ overnight.

Similarly, after synthesis of HA-IR825, $94 \mathrm{mg}$ sulfoNHS and $32 \mathrm{mg}$ EDC. $\mathrm{HCl}$ were added in the above HAIR825 suspension for $1 \mathrm{~h}$ to activate the unreacted carboxyl groups of HA-IR825. Then, $12.5 \mathrm{mg}$ Chol-PEG$\mathrm{NH}_{2}$ dissolved in a small amount of DMSO was added into the above suspension and the mixture was stirred at room temperature in the dark for $24 \mathrm{~h}$. Finally, the mixture was dialyzed sequentially against "DMSO + deionized water" and deionized water using a dialysis membrane (MWCO $=5000$ ) overnight to obtain HAIR825-Chol. Additionally, HA-IR825-mPEG was prepared with the same procedure except that the $12.5 \mathrm{mg}$ Chol-PEG- $\mathrm{NH}_{2}$ was replaced by $10.9 \mathrm{mg}$ mPEG-NH${ }_{2}$.

\section{Synthesis of HA-IR825-Chol/HCPT}

After the synthesis of HA-IR825-Chol, $10 \mathrm{mg}$ HCPT dissolved in a small amount of DMSO was added into the HA-IR825-Chol suspension. The mixed suspension was subjected to tip sonication for $5 \mathrm{~min}$ (25\% of the maximal output, $3 \mathrm{~s}$-pulse on, $3 \mathrm{~s}$-pulse off), and then stirred at 


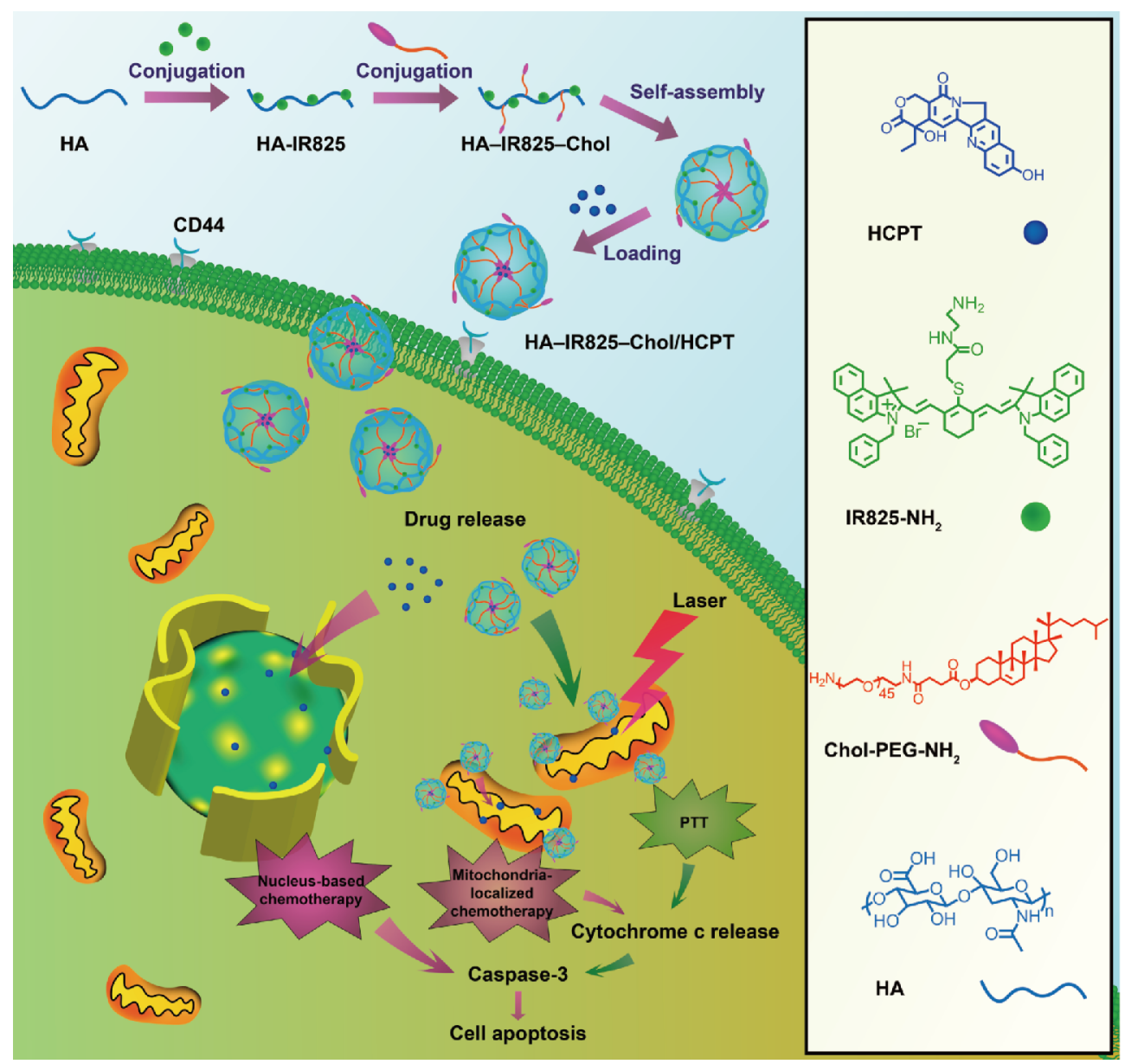

Scheme 1 Schematic illustrating the preparation of HA-IR825-Chol/HCPT NPs and their effects on cancer cells.

room temperature in the dark for $24 \mathrm{~h}$. The resultant mixture was centrifuged at $5000 \mathrm{rpm}$ for 5 min to remove the precipitates formed by unencapsulated HCPT molecules and dialyzed against deionized water using a dialysis membrane $(\mathrm{MWCO}=5000)$ for $24 \mathrm{~h}$ to obtain HAIR825-Chol/HCPT NPs.

\section{RESULTS AND DISCUSSION}

\section{Preparation and characterization of HA-IR825-Chol NPs}

The HA-IR825 NPs were prepared by conjugating HA with IR825-NH $\mathrm{N}_{2}$. Next, HA-IR825-Chol NPs were formed by reaction between HA-IR825 and Chol-PEG-NH $\mathrm{N}_{2}$. The successful synthesis of HA-IR825-Chol NPs was confirmed by Fourier-transform infrared (FTIR) spectroscopy (Fig. S1). The two peaks at 1616 and $842 \mathrm{~cm}^{-1}$ in the FTIR spectrum of HA-IR825-Chol come from the remaining carboxyl groups of the HA moieties and the PEG segment, indicating the successful conjugation be- tween Chol-PEG- $\mathrm{NH}_{2}$ and HA. We also determined the molar ratio of HA:IR825 (from IR825- $\mathrm{NH}_{2}$ ): Chol (from Chol-PEG- $\mathrm{NH}_{2}$ ) is 1:36:23 by using weighing and ultraviolet-visible (UV-vis) spectroscopy.

Next, transmission electron microscopy (TEM) was utilized to characterize the size and morphology of HAIR825-Chol dispersed in water. The TEM image in Fig. 1a demonstrated the formation of uniform nanospheres of HA-IR825-Chol with an average size of $\sim 43.5 \mathrm{~nm}$. The hydrodynamic size of HA-IR825-Chol NPs in a phosphate-buffered saline (PBS) solution was $64.9 \pm 2.9 \mathrm{~nm}$, as measured by dynamic light scattering (DLS), which was slightly larger than that $(62.5 \pm 3.2 \mathrm{~nm})$ of HA-IR825 (Fig. 1b), suggesting the successful conjugation of CholPEG- $\mathrm{NH}_{2}$ with HA-IR825 to form HA-IR825-Chol. The zeta potential values of HA-IR825 and HA-IR825-Chol were measured to be $-34.7 \pm 1.4$ and $-23.9 \pm 1.9 \mathrm{mV}$, respectively (Fig. 1c). The altered zeta potential of HAIR825-Chol compared with that of HA-IR825 indicated 
$\mathbf{a}$
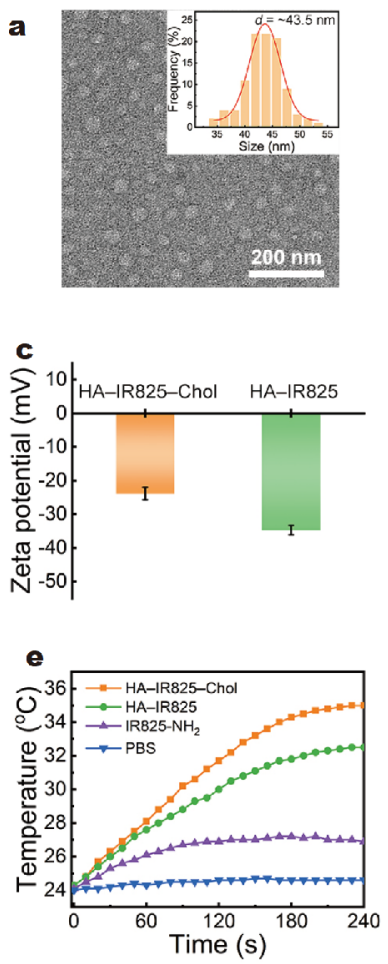

Figure 1 (a) TEM image and corresponding size distribution histogram (inset) of HA-IR825-Chol NPs. Scale bar $=200 \mathrm{~nm}$. (b) DLS results and (c) zeta potentials of HA-IR825-Chol and HA-IR825 NPs in PBS solutions. (d) UV-vis absorption spectra of IR825- $\mathrm{NH}_{2}$ (in PBS and DMSO), HA-IR825 (in PBS), and HA-IR825-Chol (in PBS) at the same IR825 concentration $\left(5 \mu \mathrm{g} \mathrm{mL}^{-1}\right)$. (e) Photothermal heating curves of IR825$\mathrm{NH}_{2}$, HA-IR825, and HA-IR825-Chol at the same IR825 concentration $\left(5 \mu \mathrm{g} \mathrm{mL}^{-1}\right)$ under the continuous $808 \mathrm{~nm}$ laser irradiation $\left(1.0 \mathrm{~W} \mathrm{~cm}^{-2}\right)$ for $4 \mathrm{~min}$. PBS was set as the control group. (f) Plots of relative absorbance values versus time for evaluating the photostability of IR825- $\mathrm{NH}_{2}$, HA-IR825, and HA-IR825-Chol at the same IR825 concentration $\left(5 \mu \mathrm{g} \mathrm{mL}^{-1}\right)$.

the consumption of the carboxyl groups by Chol-PEG$\mathrm{NH}_{2}$. Further, the UV-vis absorption spectra of IR825$\mathrm{NH}_{2}$ (dissolved in DMSO and PBS), HA-IR825 (dispersed in PBS), and HA-IR825-Chol (dispersed in PBS) were measured. It was observed that the absorption peak of IR825- $\mathrm{NH}_{2}$ in DMSO was much sharper than that in the PBS solution (Fig. 1d), indicating the better dispersity of the dye in DMSO. Similarly, the sharper absorption band of HA-IR825-Chol than that of HA-IR825 suggested the better dispersity of HA-IR825-Chol, as proved by its smaller polydispersity index (PDI) (Fig. 1b).

To investigate whether HA-IR825-Chol NPs possess desirable photothermal properties, we recorded the temperatures of PBS solution, IR825- $\mathrm{NH}_{2}$ solution, HAIR825 suspension, and HA-IR825-Chol suspension under continuous laser irradiation at $808 \mathrm{~nm}$ for $4 \mathrm{~min}$ (Fig. 1e). The results revealed that the temperature elevations of the three samples were ranked as follows: HA-IR825-Chol > HA-IR825 > IR825- $\mathrm{NH}_{2}$, suggesting the improved photothermal conversion ability of HA-IR825-Chol compared with the other two samples. Furthermore, the photostabilities of IR825- $\mathrm{NH}_{2}, \mathrm{HA}-\mathrm{IR} 825$, and HAIR825-Chol were also investigated via UV-vis spectroscopy (Figs S2-S4), and the calculated relative absorbance values were presented in Fig. 1f. The photodegradation process of IR825 moieties in HA-IR825 or HA-IR825Chol NPs was remarkably slowed down. The results showed that HA-IR825 and HA-IR825-Chol NPs own better photostability than IR825- $\mathrm{NH}_{2}$, indicating the protective role of HA and Chol-PEG moieties. In addition, the photothermal conversion efficiencies of IR825$\mathrm{NH}_{2}$ and HA-IR825-Chol were calculated to be $16.0 \%$ and $24.3 \%$, respectively, according to a previously reported method $[20,49,50]$, revealing the improved photothermal property of HA-IR825-Chol compared with that of IR825- $\mathrm{NH}_{2}$.

\section{Cellular uptake behavior and cytotoxicity of HA-IR825- Chol NPs}

Using murine mammary cancer cells ( $4 \mathrm{~T} 1$ cells) as the model cells, we then investigated the cellular uptake behaviors of IR825- $\mathrm{NH}_{2}$, HA-IR825, and HA-IR825-Chol by using confocal microscopy and flow cytometry. Meanwhile, to prove the effect of Chol on the cellular internalization of HA-IR825-Chol, we also synthesized HA-IR825-poly(ethylene glycol) ${ }_{2 \mathrm{k}}$-methoxy (termed HAIR825-mPEG) by conjugating HA-IR825 with methoxypoly(ethylene glycol $)_{2 \mathrm{k}}-\mathrm{NH}_{2}\left(\mathrm{mPEG}-\mathrm{NH}_{2}\right)$ and evaluated its cellular uptake behavior. As shown in Fig. 2a and c, HA-IR825-Chol exhibited the largest cellular uptake efficiency among all the groups, showing the crucial role of Chol in the cellular internalization of HA-IR825-Chol. Besides, we investigated the intracellular location of HAIR825-Chol by co-staining the 4T1 cells with HA-IR825Chol and one of the following commercially available organelle-selective trackers: rhodamine 123 (Rhod 123, a commercial mitochondrial dye), ER-Tracker Green (ERTracker, for staining endoplasmic reticulum, ER), GolgiTracker Green (Golgi-Tracker, for staining Golgi apparatus), and LysoTracker Green (LysoTracker, for staining lysosomes). Fig. $2 \mathrm{~b}$ revealed that the green fluorescence of Rhod 123 well overlapped with the red fluorescence of the IR825 in HA-IR825-Chol, and the corresponding Pearson's correlation coefficient (PCC) and overlap coefficient (OLC) were 0.98 and 0.98 , respectively. In contrast, HAIR825-Chol did not stain ER well, and the corresponding PCC and OLC were 0.74 and 0.74 , respectively. Further, a 


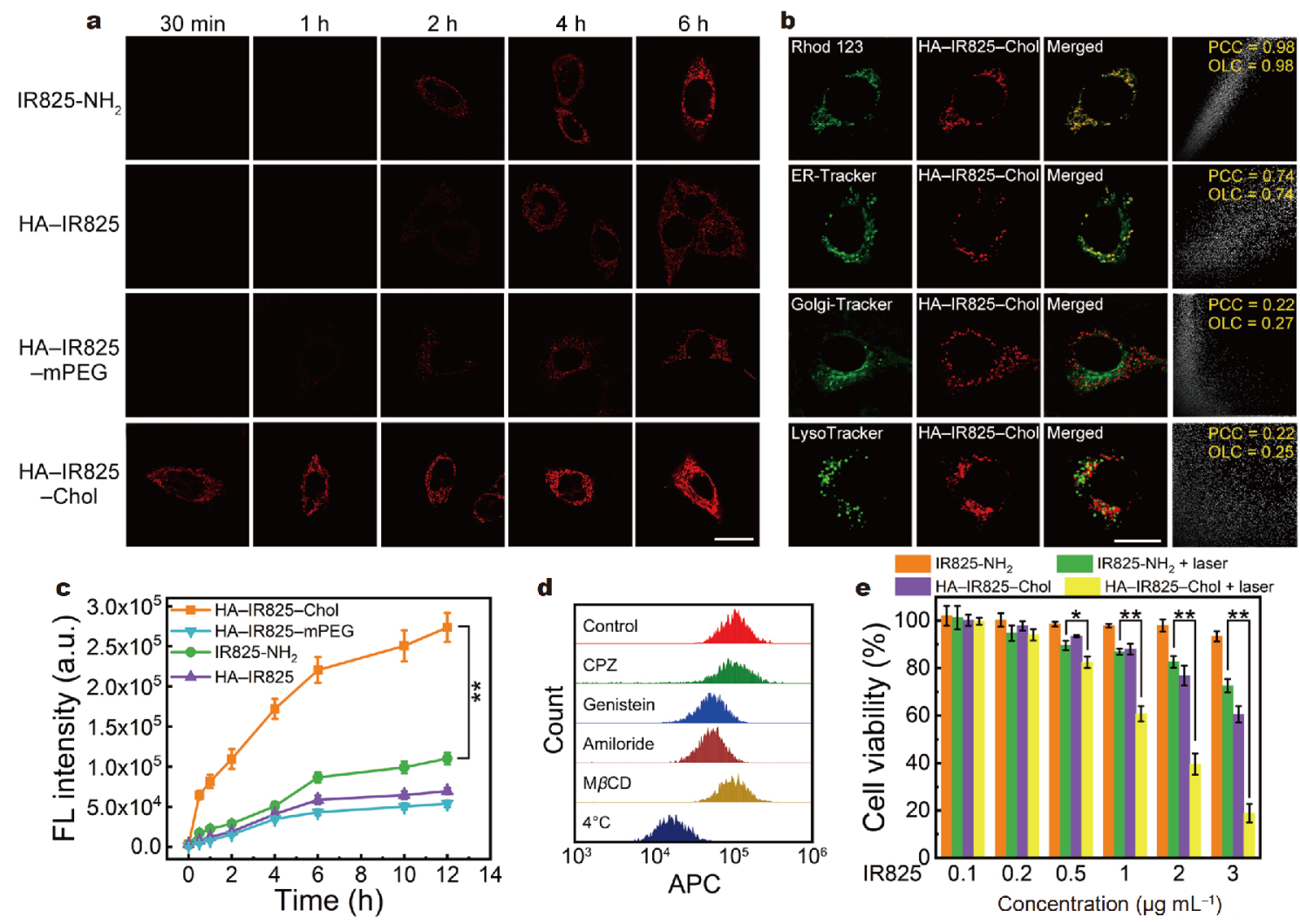

Figure 2 (a) Confocal fluorescence images of $4 \mathrm{~T} 1$ cells after incubation with IR825-NH $\mathrm{N}_{2}$, HA-IR825, HA-IR825-mPEG, or HA-IR825-Chol $\left(2 \mu \mathrm{g} \mathrm{mL}^{-1}\right.$ IR825 in each sample) for different time periods. Scale bar $=20 \mu \mathrm{m}$. (b) Confocal fluorescence images of 4T1 cells incubated with HAIR825-Chol $\left(2 \mu \mathrm{g} \mathrm{mL}^{-1}\right.$ of IR825) for $2 \mathrm{~h}$ and then stained by Rhod $123\left(100 \mathrm{nmol} \mathrm{L}{ }^{-1}\right)$ for $10 \mathrm{~min}$, ER-Tracker $\left(1 \mu \mathrm{mol} \mathrm{L}{ }^{-1}\right)$ for $30 \mathrm{~min}$, Golgi-Tracker $\left(5 \mu \mathrm{mol} \mathrm{L}^{-1}\right)$ for $30 \mathrm{~min}$, or LysoTracker $\left(1 \mu \mathrm{mol} \mathrm{L}^{-1}\right)$ for $30 \mathrm{~min}$. Scale bar $=20 \mu \mathrm{m}$. (c) Fluorescence (FL) intensities of $4 \mathrm{~T} 1$ cells after incubation with IR825- $\mathrm{NH}_{2}$, HA-IR825, HA-IR825-mPEG, or HA-IR825-Chol $\left(2 \mu \mathrm{g} \mathrm{mL}{ }^{-1}\right.$ IR825 in each sample) for different time periods as measured by flow cytometry. (d) Effects of various endocytosis inhibitors and low temperature $\left(4^{\circ} \mathrm{C}\right)$ on the endocytosis of HA-IR825-Chol by $4 \mathrm{~T} 1$ cells evaluated via flow cytometry by using the allophycocyanin (abbreviated as APC in the figure) channel. (e) Relative viabilities of $4 \mathrm{~T} 1$ cells after various treatments. ${ }^{*} P<0.05,{ }^{* *} P<0.01$.

poor overlap was seen in the cases of Golgi apparatus and lysosome, with a PCC of 0.22 and 0.22 , respectively. These results suggested that HA-IR825-Chol has an excellent mitochondrial targeting capability, which may possibly be attributed to the excellent mitochondrial targeting property of IR 825 .

Furthermore, we investigated the cell endocytic pathways of HA-IR825-Chol by using 4T1 cells. Chlorpromazine (CPZ) hydrochloride, genistein, 5- $(N, N-$ dimethyl)-amiloride (amiloride), methyl- $\beta$-cyclodextrin $(\mathrm{M} \beta \mathrm{CD})$, and $4^{\circ} \mathrm{C}$ treatment were used to inhibit clathrinmediated, caveolae-mediated, macropinocytosis-dependent, lipid raft-mediated, and energy-dependent pathway, respectively [69]. The flow cytometric results in Fig. 2d and Fig. S5 showed that genistein, amiloride, and $4^{\circ} \mathrm{C}$ treatment evidently decreased the internalization of HAIR825-Chol, indicating that the HA-IR825-Chol NPs were internalized mainly through caveolae-mediated, macropinocytosis- and energy-dependent endocytosis.

Meanwhile, the dark toxicity and phototoxicity of HAIR825-Chol were tested by using 4T1 cells. The results in Fig. 2e indicated that free IR825- $\mathrm{NH}_{2}$ did not elicit evident dark toxicity to the cells at the tested concentrations, while HA-IR825-Chol NPs exhibited significantly higher phototoxicity than IR825- $\mathrm{NH}_{2}$ due to their remarkable endocytosis by cancer cells.

\section{Characterization and cellular experiments of HA-IR825- Chol/HCPT NPs}

We next explored the feasibility of using the HA-IR825Chol NPs as the nanocarriers of chemotherapeutics. HCPT, a DNA topoisomerase I inhibitor [70,71], has extensively entered clinical trials; however, its extremely poor water-solubility severely hampers the efficient nu- 
clear delivery of the drug. Because HCPT owns an excellent ability to act on DNA in the nucleus and mitochondria [72], we anticipate that the HCPT-loaded HAIR825-Chol NPs (termed HA-IR825-Chol/HCPT NPs) can realize combined chemo-photothermal therapy for improving the final therapeutic outcomes.

The as-prepared HA-IR825-Chol/HCPT NPs displayed an average size of $65.5 \pm 3.5 \mathrm{~nm}$ and their zeta potential was measured to be $-18.6 \pm 1.0 \mathrm{mV}$ (Fig. 3a and Fig. S6). The encapsulation ratio of HCPT and drug (i.e., HCPT) loading coefficient of HA-IR825-Chol/HCPT NPs were $8.1 \%$ and $0.7 \%$, respectively. In addition, we measured the UV-vis absorption spectra of HCPT and HA-IR825$\mathrm{Chol} / \mathrm{HCPT}$ in PBS $\left(10 \mathrm{mmol} \mathrm{L}^{-1}, \mathrm{pH}\right.$ 7.4) containing $0.1 \%$ Triton X-100 solution (to dissolve HCPT) and HAIR825-Chol in PBS (Fig. 3b). The UV-vis results showed that HA-IR825-Chol/HCPT (obtained after centrifugation and dialysis) dispersed in PBS containing $0.1 \%$ Triton $\mathrm{X}-100$ had a new absorption peak at $377 \mathrm{~nm}$ (a characteristic peak of HCPT) relative to HA-IR825-Chol, indicating the presence of HCPT in the nanocomplexes. Further, the release kinetics of HCPT from HA-IR825$\mathrm{Chol} / \mathrm{HCPT} \mathrm{NPs}$ at $\mathrm{pH} 6.5$ (tumor microenvironment condition) and $\mathrm{pH} 7.4$ (normal tissue condition) were evaluated (Fig. S7). The results indicated that the nanoagents have a similar drug release behavior in slightly acidic and neutral solutions.

Next, the subcellular fate of HA-IR825-Chol/HCPT NPs after cellular internalization was studied by confocal imaging. In Fig. 3c, the HCPT loaded in HA-IR825-Chol NPs showed enhanced cellular uptake compared with free HCPT in $4 \mathrm{~T} 1$ cells. Besides, it could be seen that the HCPT in HA-IR825-Chol/HCPT NPs gradually entered the nuclei as the incubation time increased. We also found that the fluorescence signals of HCPT partially matched those of Rhod 123 (Fig. 3d), suggesting the mitochondrial accumulation of HCPT. The two main pathways of chemotherapy-induced tumor cell apoptosis include the mitochondrial pathway and the death receptor pathway [73]. The mitochondrial membrane potential of cells reflects the degree of mitochondrial apoptosis. Hence, to investigate the effect of HA-IR825$\mathrm{Chol} / \mathrm{HCPT}$ on the mitochondrial pathway, the mitochondrial membrane potentials of the cells with different treatments (i.e., control (untreated), HCPT, and HA-IR825-Chol/HCPT) were compared via measuring the fluorescence intensities of Rhod 123 for $4 \mathrm{~T} 1$ cells. The results in Fig. 3e showed that HA-IR825-Chol/HCPT could more significantly affect the mitochondrial membrane potential of the cancer cells compared with free
HCPT and the control group, and free HCPT did not cause an evident alteration of the mitochondrial membrane potential of the cells relative to the control group. These results indicated that HA-IR825-Chol/HCPT achieved an enhanced apoptosis-inducing effect on the mitochondrial pathway of the cancer cells.

In addition, to further explore the impact of HA-IR825Chol/HCPT NPs on mitochondria, we carried out immunofluorescence staining to visualize cytochrome $c$ in the $4 \mathrm{~T} 1$ cells. Cytochrome c, as an indicator of apoptosis, is located at the mitochondrial inner membrane and cannot penetrate through the outer membrane under normal conditions, while it is released into the cytoplasm during apoptosis as a consequence of the altered mitochondrial membrane permeability [72,74-76]. Rabbit anti-cytochrome $c$ antibody (combined with fluorescein isothiocyanate (FITC)-labeled goat anti-rabbit IgG antibody) and Hoechst 33342 were used to stain cytochrome $c$ and cell nuclei, respectively. As shown in Fig. $3 \mathrm{f}$ and Fig. S8, the "HA-IR825-Chol/HCPT + laser" group showed the strongest green fluorescence signals in the cytosol compared with other groups, suggesting that cytochrome c was released into the cytosol from the mitochondrial membrane. Meanwhile, the "HA-IR825-Chol/HCPT" and "HA-IR825-Chol + laser" groups also showed stronger green fluorescence signals in the cytosol than other groups (except the "HA-IR825-Chol/HCPT + laser" group), indicating that after being encapsulated by HAIR825-Chol, HCPT could destroy the structure of mitochondria and the mitochondria-targeted HA-IR825Chol could damage the mitochondria with the help of laser irradiation. The above results might indicate that, compared with other groups, HA-IR825-Chol/HCPT plus laser irradiation achieved the highest anticancer efficacy, possibly due to their strongest capability to induce cell apoptosis, as evidenced by the most evident cytochrome $\mathrm{c}$ release from mitochondria.

Further, to elucidate the death receptor pathway mechanism of cancer cells induced by various treatments, we investigated the cleaved caspase- 3 expression in $4 \mathrm{~T} 1$ cells via immunofluorescence staining. Caspase- 3 is a frequently activated death protease, catalyzing the specific cleavage of many key cellular proteins. Caspase- 3 is seen as a crucial mediator of programmed cell death and a typical hallmark of apoptosis [74]. More importantly, the pathway of caspase- 3 activation was found to be dependent on mitochondrial cytochrome $c$ release [77], and HCPT acting on nuclear DNA was demonstrated to trigger the upregulation of cleaved caspase-3 [78]. The results in Fig. $3 \mathrm{~g}$ and Fig. S9 revealed that the fluores- 

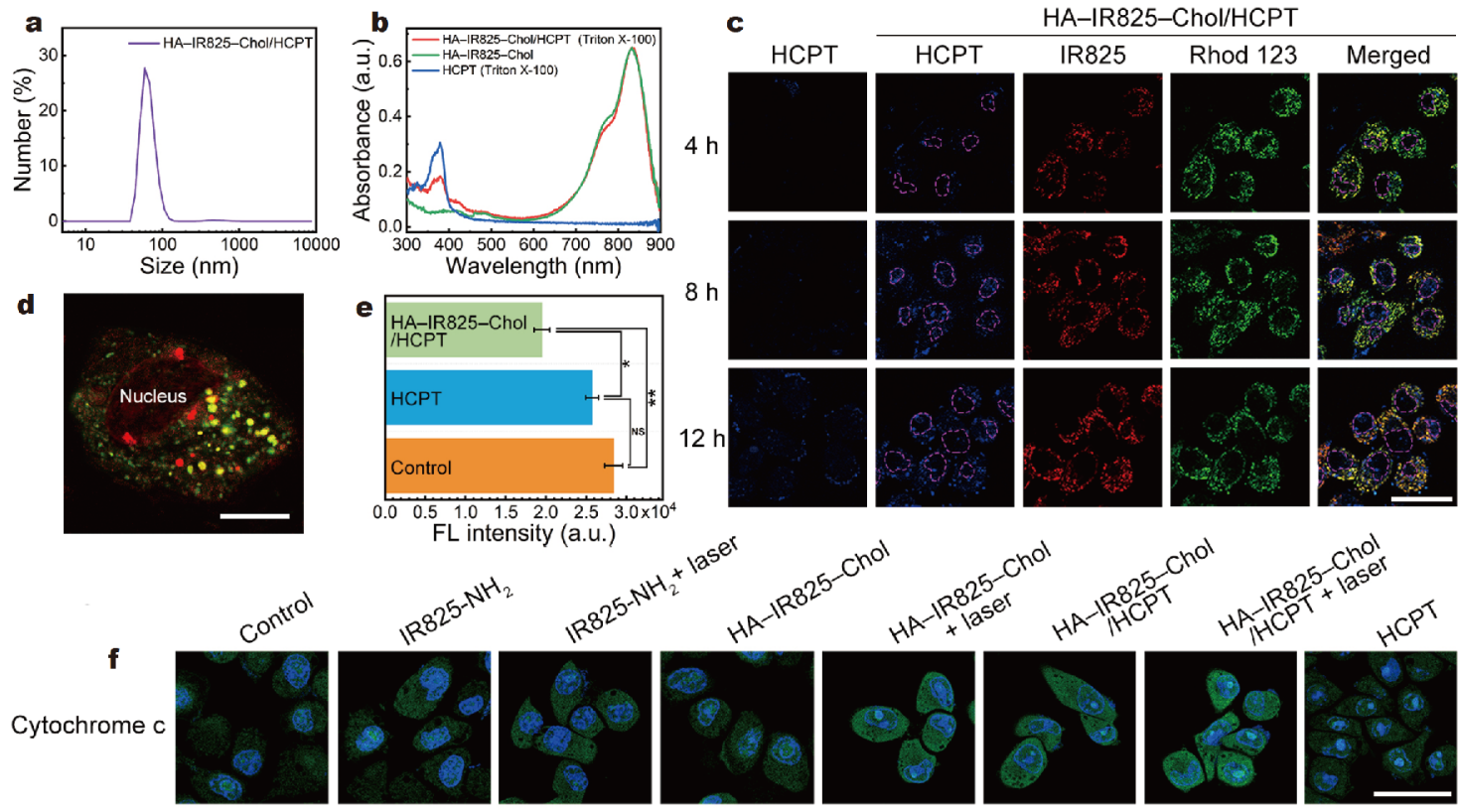

$88^{25}{ }^{-N \mathrm{NH}_{2}}$<smiles>C1C[C@H]2CC[C@H]1C2</smiles><smiles>C1CCCCC1</smiles>
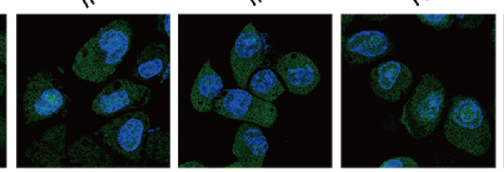<smiles>C1C2CC3CC1C3C2</smiles><smiles>C1C[C@H]2OC[C@H]12</smiles><smiles>C1C[As]2CCC2[As]1</smiles>
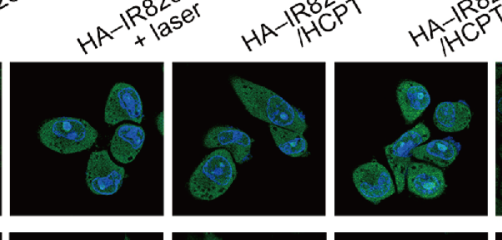

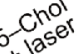

$\mathbf{g}$
Cleaved
caspase-3
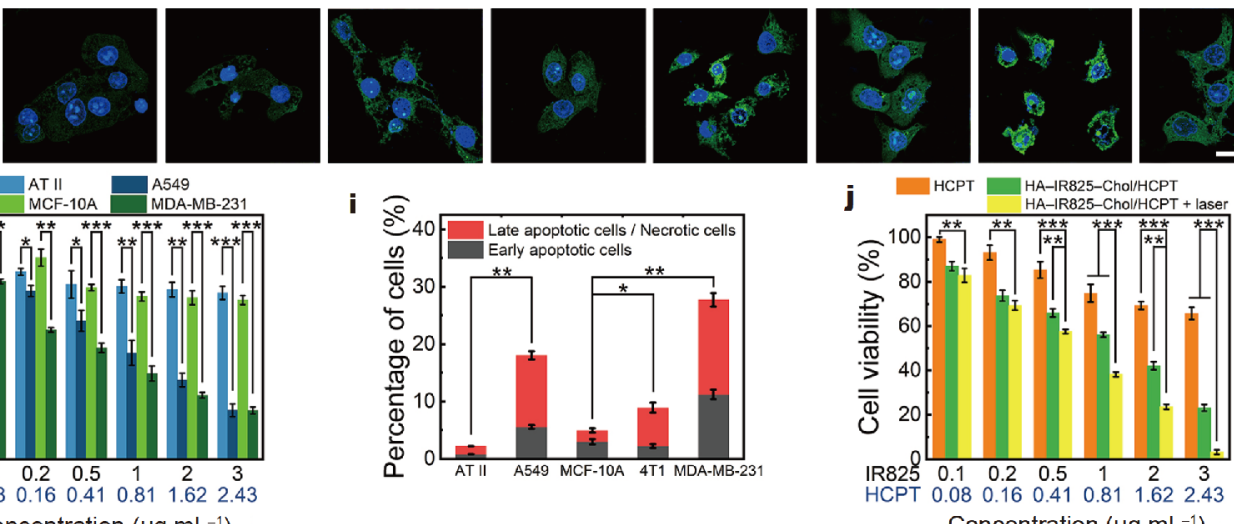

h $\begin{array}{ll}\text { AT II } & \text { A549 } \\ \text { MCF-10A } & \text { MDA-MB-231 }\end{array}$
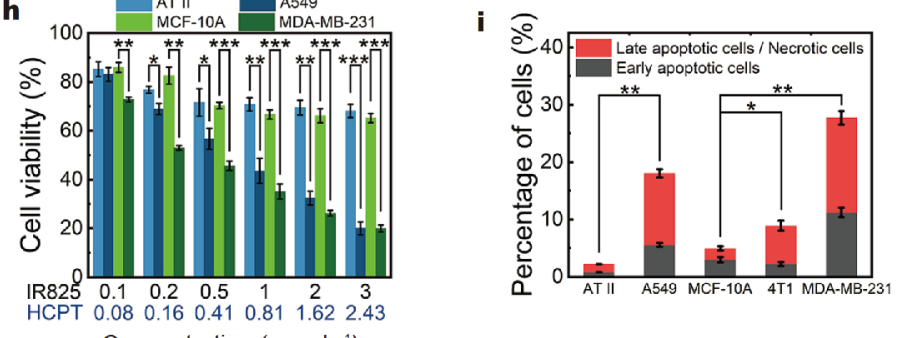

Concentration $\left(\mu \mathrm{g} \mathrm{mL}^{-1}\right)$

Concentration $\left(\mu \mathrm{g} \mathrm{mL} \mathrm{L}^{-1}\right)$

Figure 3 (a) Hydrodynamic diameter of HA-IR825-Chol/HCPT NPs in a PBS solution (pH 7.4). (b) UV-vis absorption spectra of HCPT (in PBS with $0.1 \%$ Triton X-100), HA-IR825-Chol (in PBS), and HA-IR825-Chol/HCPT (in PBS with $0.1 \%$ Triton X-100). (c) Confocal fluorescence images of $4 \mathrm{~T} 1$ cells after incubation with HCPT or HA-IR825-Chol/HCPT NPs at the same HCPT concentration of $1.6 \mu \mathrm{mL}^{-1}$ for different time periods. Before imaging, the HA-IR825-Chol/HCPT-treated cells were stained by Rhod 123 to visualize mitochondria. The dotted circles in purple indicate the positions of nuclei. Scale bar $=25 \mu \mathrm{m}$. (d) Confocal fluorescence image of a representative 4T1 cell with the same treatment as that in (c). The red color (pseudo-color) stands for the fluorescence of HCPT and the green color stands for the fluorescence of Rhod 123. The yellow color, the merged color of the red and green colors, indicates the colocalization of HCPT and mitochondrion. Scale bar $=10 \mu \mathrm{m}$. (e) FL intensities of Rhod 123 (as measured by flow cytometry) in $4 \mathrm{~T} 1$ cells after different treatments as indicated. Rhod 123 was added to the treated cells to reflect the mitochondrial membrane potentials of the cells. (f, g) Immunofluorescence staining results of $4 \mathrm{~T} 1$ cells with different treatments as indicated. IR825-NH 2 , HAIR825-Chol, HA-IR825-Chol/HCPT: $2 \mu \mathrm{g} \mathrm{mL}^{-1}$ of IR825. HCPT: $1.6 \mu \mathrm{g} \mathrm{mL}^{-1}$. Laser irradiation: $808 \mathrm{~nm}, 1.0 \mathrm{~W} \mathrm{~cm}{ }^{-2}, 5 \mathrm{~min}$. For (f), before imaging, the treated cells were stained by "rabbit anti-cytochrome c antibody + FITC-labeled goat anti-rabbit IgG antibody" and Hoechst 33342 (abbreviated as Hoechst in the figure) to visualize cytochrome $\mathrm{c}$ and cell nuclei, respectively. Scale bar $=25 \mu \mathrm{m}$. For (g), before imaging, the treated cells were stained by "cleaved caspase-3 rabbit monoclonal antibody + FITC-labeled goat anti-rabbit IgG antibody" and Hoechst 33342 (abbreviated as Hoechst in the figure) to visualize cleaved caspase-3 and cell nuclei, respectively. Scale bar $=25 \mu \mathrm{m}$. (h) Relative viabilities of AT II, A549, MCF-10A, and MDA-MB231 cells after treatment with different concentrations (based on IR825 or HCPT) of HA-IR825-Chol/HCPT NPs for $24 \mathrm{~h}$. (i) Statistical results (obtained via flow cytometry) of the early apoptotic and late apoptotic/necrosis cells for different cell lines. Before flow cytometry analysis, the cells were incubated with HA-IR825-Chol/HCPT $\left(0.5 \mu \mathrm{g} \mathrm{mL}{ }^{-1}\right.$ of IR825) for $24 \mathrm{~h}$. (j) Relative viabilities of $4 \mathrm{~T} 1$ cells after various treatments. ${ }^{*} P<0.05,{ }^{* *} P<$ $0.01,{ }^{* *} P<0.001$, ns: nonsignificant difference.

cence signals from the immunofluorescence-labeled cleaved caspase-3 in the HA-IR825-Chol/HCPT group were stronger than those in the HA-IR825-Chol and HCPT groups, implying that HA-IR825-Chol/HCPT 
could exert a stronger apoptosis-inducing effect. The "HA-IR825-Chol/HCPT + laser" led to the highest cleaved caspase-3 level compared with other groups, indicating that the combined chemo-photothermal therapy could result in a higher level of cell apoptosis.

We then evaluated the toxicity of HA-IR825-Chol/ HCPT toward different cell types via 3-(4,5-dimethyl-2thiazolyl)-2,5-diphenyl-2H-tetrazolium bromide (MTT) assay. The results in Fig. $3 \mathrm{~h}$ revealed that the viabilities of human normal lung (AT II) cells were evidently higher than those of human lung cancer (A549) cells after treatment with the respective concentrations of HAIR825-Chol/HCPT. The higher cytotoxicity of the NPs to the A549 cells might be due to the higher uptake amount of the NPs in the A549 cells than that in the AT II cells (Fig. S10). Similarly, the NPs also elicited higher toxicities to the human breast cancer (MDA-MB-231) cells than the human normal breast (MCF-10A) cells. The selective cancer cell killing activity of the NPs indicated that the HA-containing NPs could preferentially target the CD44overexpressed cancer cells via HA-CD44 recognition. To prove this, $4 \mathrm{~T} 1$ cells were pretreated with the HA polymers $\left(50 \mu \mathrm{g} \mathrm{mL}^{-1}, 2 \mathrm{~h}\right)$ to block the HA receptors, followed by HA-IR825-Chol incubation. The results showed that the cellular uptake of the NPs was significantly weakened (Fig. S11), which further validated the HAendowed cancer cell targeting property of HA-IR825Chol.

In addition, we further performed flow cytometric analysis to confirm the above MTT results via the annexin V-FITC/propidium iodide (PI) dual staining assay. In Fig. 3i, the rate of apoptotic cells (early and late apoptotic cells) and necrotic cells in the AT II group was lower than that in the A549 group. Similarly, the rate of apoptotic and necrotic cells in the MCF-10A group was lower than that in the $4 \mathrm{~T} 1$ or MDA-MB-231 group. These results confirmed that the $\mathrm{HA}-\mathrm{CD} 44$ recognition played an important role in inducing a higher toxicity of the NPs to the cancer cells. Then, the in vitro anticancer efficacy of HA-IR825-Chol/HCPT was studied by the MTT assay (Fig. 3j). Compared with free HCPT, HA-IR825-Chol/ HCPT showed significantly higher dark cytotoxicity at respective HCPT concentrations, indicating that the significantly enhanced HCPT internalization of HA-IR825$\mathrm{Chol} / \mathrm{HCPT}$ relative to the internalization of free HCPT (as revealed in Fig. 3c) was responsible for the higher cytotoxicity of the NPs. In addition, "HA-IR825-Chol/ HCPT + laser" group displayed higher toxicity than the other groups, demonstrating that the combined chemophotothermal therapy could achieve the best cancer therapeutic outcome.

\section{In vivo fluorescence imaging and combination cancer therapy}

The in vivo biodistribution of the HA-IR825-Chol/HCPT NPs in $4 \mathrm{~T} 1$ tumor-bearing nude mice was monitored using an in vivo fluorescence imaging system. As displayed in Fig. $4 \mathrm{a}$ and b, the HA-IR825-Chol/HCPT NPs significantly accumulated in the tumor region at $1 \mathrm{~h}$ after intravenous injection of the NPs and achieved stable tumor retention for at least $24 \mathrm{~h}$. In contrast, negligible fluorescence signals could be observed at the tumor site in the free IR825- $\mathrm{NH}_{2}$ group during the observation period. Further, the ex vivo fluorescence imaging of the major organs and tumor tissues of the mice sacrificed at 1,3 , and $7 \mathrm{~d}$ after the intravenous injection of free IR825- $\mathrm{NH}_{2}$ or HA-IR825-Chol/HCPT was performed. The results in Fig. $4 \mathrm{c}$ and $\mathrm{d}$ revealed that, for free IR825- $\mathrm{NH}_{2}$ molecules, they were mainly in the liver, spleen, lung, and kidneys at $1 \mathrm{~d}$ postinjection, and at $3 \mathrm{~d}$ postinjection, the liver deposition of the molecules became even higher, accompanied by the decreased accumulation in the lung and kidneys; for the HA-IR825-Chol/HCPT NPs, they were distributed in all the major organs (except the heart) and tumor tissue at $1 \mathrm{~d}$ postinjection, and at $3 \mathrm{~d}$ postinjection, evident accumulation in the liver and tumor could still be observed. In addition, it was found that for the HAIR825-Chol/HCPT NPs, they could be cleared out of the mouse body after 7 days, with negligible fluorescence signals in the major organs and tumor; while for the free IR825- $\mathrm{NH}_{2}$ molecules, evident fluorescence signals could still be seen in the liver and spleen after 7 days. The efficient body clearance of the HA-IR825-Chol/HCPT NPs might ensure the in vivo biosafety of the nanoagents.

To carry out in vivo PTT treatments, we first monitored the temperature changes of the tumors of the mice after intravenous injection of PBS, IR825- $\mathrm{NH}_{2}$, and HA-IR825Chol/HCPT under laser irradiation $(808 \mathrm{~nm}$, $1.0 \mathrm{~W} \mathrm{~cm}^{-2}$ ). The results in Fig. $4 \mathrm{e}$ and $\mathrm{f}$ showed that after irradiation for $10 \mathrm{~min}$, the tumor temperatures of the mice in the PBS, IR825- $\mathrm{NH}_{2}$, and HA-IR825-Chol/HCPT groups reached $34.0,38.4$, and $46.2^{\circ} \mathrm{C}$.

Meanwhile, to evaluate the antitumor efficiencies of various treatments in $4 \mathrm{~T} 1$ tumor-bearing $\mathrm{BALB} / \mathrm{c}$ nude mice, we carried out hematoxylin and eosin (H\&E) staining experiments, and the results shown in Fig. $4 \mathrm{~g}$ demonstrated the severest damage to the tumor tissue after the "HA-IR825-Chol/HCPT + laser" treatment, which was in good accordance with its strongest tumor ablation activity as shown in Fig. $4 \mathrm{~h}$. 

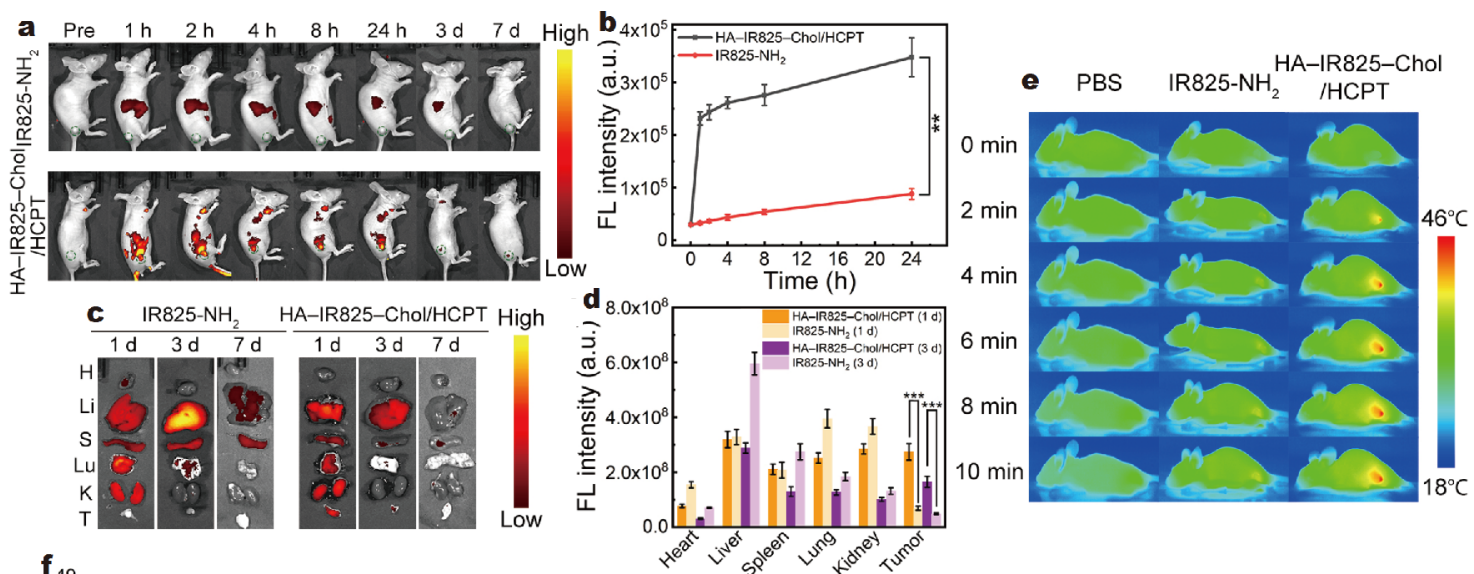
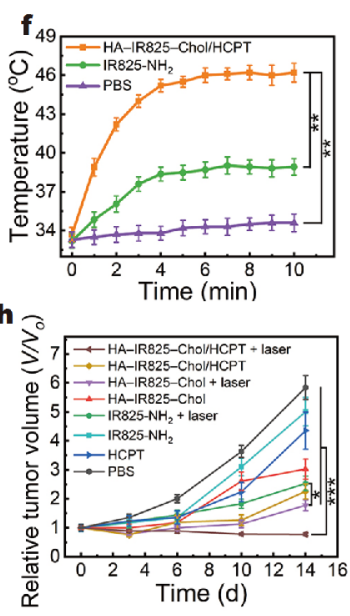

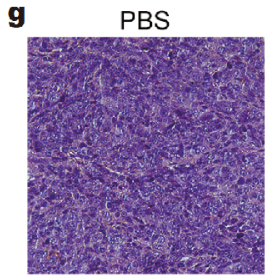

IR825- $\mathrm{NH}_{2}$

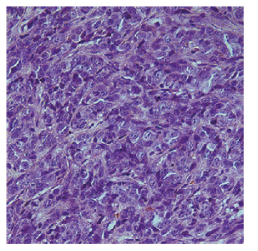

HCPT

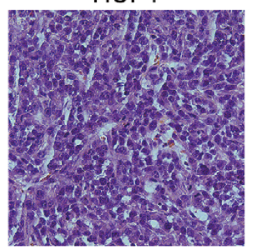

IR825- $\mathrm{NH}_{2}+$ laser

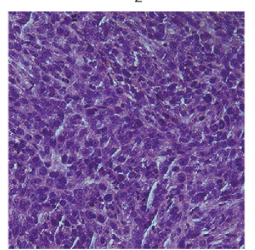

HA-IR825-Chol HA-IR825-Chol + laser

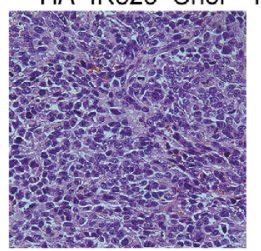

HA-IR825-Chol

/HCPT

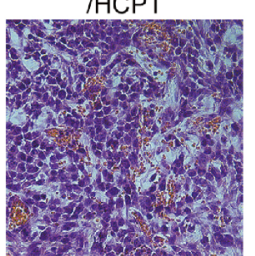

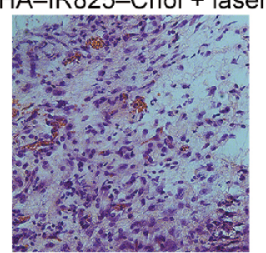

HA-IR825-Chol HCPT + laser

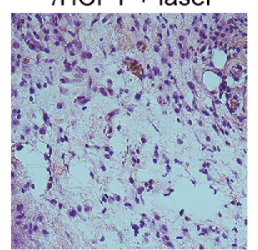

Figure 4 (a) Time-dependent in vivo fluorescence images of 4T1 tumor-bearing nude mice after intravenous injection of IR825- $\mathrm{NH}_{2}$ or HA-IR825Chol/HCPT (7 mg kg of IR825). Green dotted circles indicate the tumor sites of mice. "Pre" indicates a time point before the injection of these drugs. (b) FL intensities of the tumors partially shown in (a). (c) Ex vivo fluorescence images and (d) corresponding FL intensities of major organs and tumors excised from mice at 1,3 , and 7 (only for (c)) $\mathrm{d}$ after intravenous injection of IR825- $\mathrm{NH}_{2}$ or HA-IR825-Chol/ $\mathrm{HCPT}\left(7 \mathrm{mg} \mathrm{kg}{ }^{-1}\right.$ of IR825). (e) In vivo thermal imaging results of mice after intravenous injection of PBS, IR825- $\mathrm{NH}_{2}\left(7 \mathrm{mg} \mathrm{kg}^{-1}\right)$, and HA-IR825-Chol/ $\mathrm{HCPT}\left(7 \mathrm{mg} \mathrm{kg}{ }^{-1}\right.$ of IR825). Before imaging, the tumor sites of the mice were irradiated by a laser $\left(808 \mathrm{~nm}, 1.0 \mathrm{~W} \mathrm{~cm}^{-2}\right)$ for different time periods. (f) Temperatures of the tumor sites partially shown in (e). (g) Representative optical images of H\&E-stained tumor slices from tumor-bearing mice after various treatments. (h) Tumor growth profiles of mice after various treatments.. ${ }^{*} P<0.05,{ }^{* *} P<0.01,{ }^{* *} P<0.001$.

\section{Biosafety of HA-IR825-Chol/HCPT NPs}

Encouraged by the excellent antitumor efficiency of HAIR825-Chol/HCPT NPs, we then carefully evaluated the biosafety of the NPs in nude mice. First, no evident loss of weight was observed in all the groups with various treatments (Fig. S12). Second, the hemanalysis and blood biochemical analysis were performed for the treated mice at $14 \mathrm{~d}$, and negligible differences were observed in all the blood indexes of the control and "HA-IR825-Chol/HCPT + laser" groups (Fig. 5a-j). Meanwhile, the hemolysis results revealed that IR825- $\mathrm{NH}_{2}, \mathrm{HA}-\mathrm{IR} 825-\mathrm{Chol}$, and HA-IR825-Chol/HCPT at the highest tested concentrations of $10 \mu \mathrm{g} \mathrm{mL}^{-1}$ (based on IR825) presented ignorable hemolytic activity (Fig. 5k and Fig. S13). In addition, the H\&E staining results of the major organs of the mice in the control and "HA-IR825-Chol/HCPT + laser" groups proved no obvious systemic toxicity of the combined treatment (Fig. 5l). These results collectively indicated the good safety of the HA-IR825-Chol/HCPT NPs.

\section{CONCLUSIONS}

In this research, we designed a smart HA-IR825-Chol nanocarrier with $\mathrm{CD} 44$ recognition and mitochondriatargetable properties, which could efficiently encapsulate the chemotherapeutic drug HCPT for realizing cooperative photo-chemotherapy. It was found that, in HAIR825-Chol, the water-solubility of the modified IR825 was significantly improved, and the endocytosis rate and internalization amount of IR825 in cancer cells were considerably increased. In addition, the as-designed HA- 

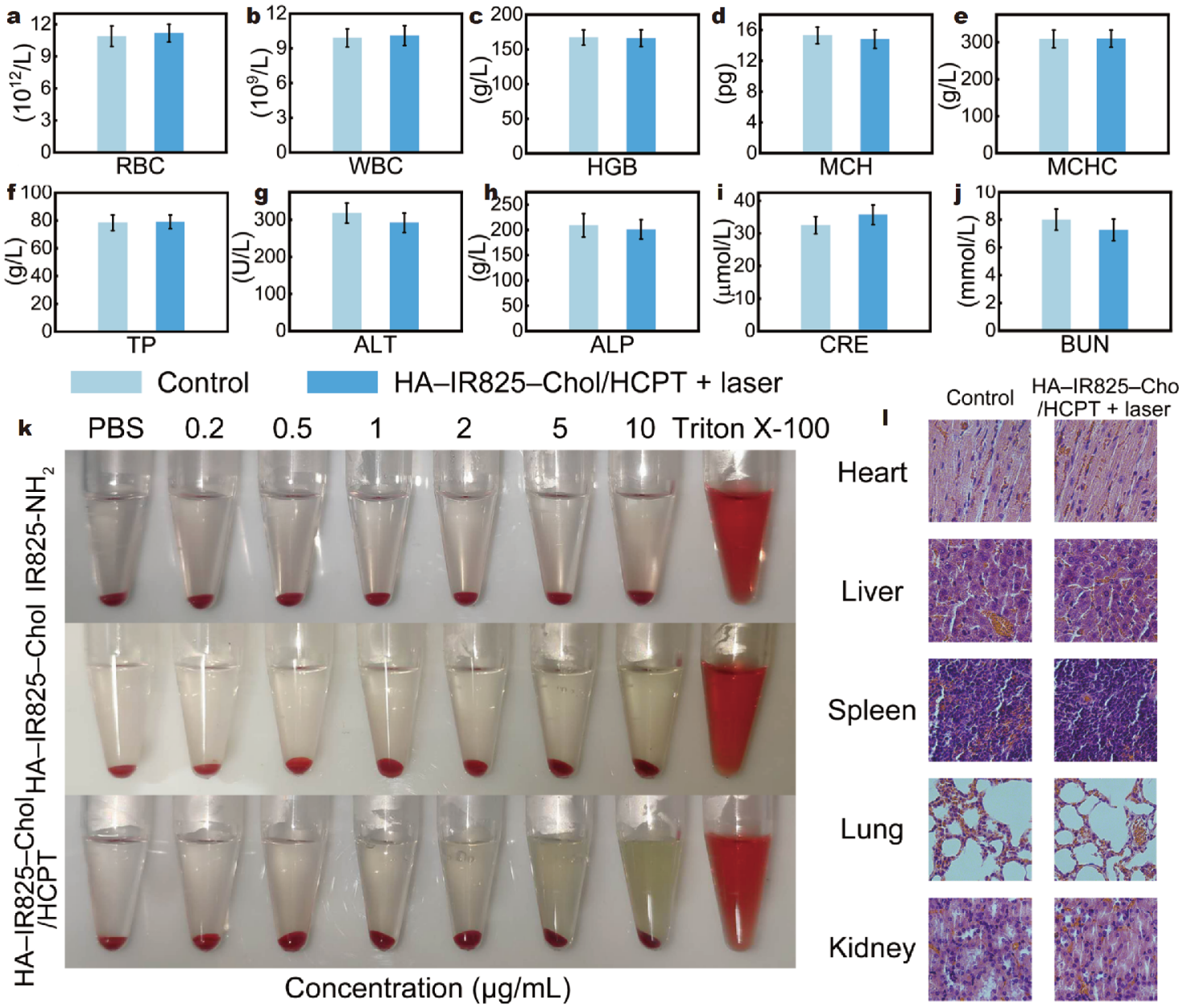

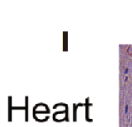

Control HA-IR825-Chol
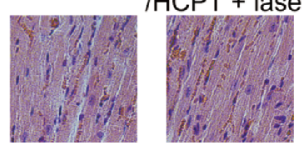

Liver
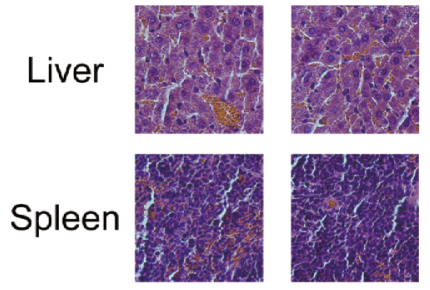

Lung
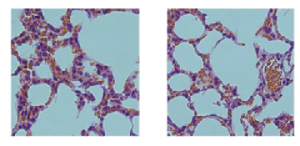

Kidney
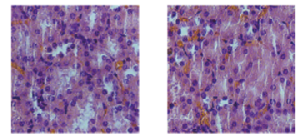

Figure $5(\mathrm{a}-\mathrm{j})$ Hemanalysis and blood biochemical analysis results collected from the healthy mice (control) or the 4T1 tumor-bearing nude mice after "HA-IR825-Chol/HCPT + laser" treatment. The tested indexes include number of red blood cells (RBC), number of white blood cells (WBC), concentration of hemoglobin (HGB), mean corpuscular hemoglobin ( $\mathrm{MCH}$ ), mean corpuscular hemoglobin concentration (MCHC), and five hepatic and renal function parameters like contents of total protein (TP), glutamate pyruvate transaminase (ALT), alkaline phosphatase (ALP), creatinine (CRE), and blood urea nitrogen (BUN). (k) Photographs of RBC suspensions after treatment with various concentrations (based on IR825) of IR825$\mathrm{NH}_{2}$, HA-IR825-Chol, or HA-IR825-Chol/HCPT. 1\% Triton X-100 solution and PBS solution were set as positive control and negative control, respectively. (1) Representative optical images of H\&E-stained slices from the major organs of a healthy mouse (control) and a tumor-bearing mouse treated with "HA-IR825-Chol/HCPT + laser".

IR825-Chol NPs owned better photothermal properties compared with IR825- $\mathrm{NH}_{2}$. Besides, by being encapsulated in the HA-IR825-Chol nanocarrier, HCPT showed significantly higher cellular uptake, and part of the HCPT molecules were located in the mitochondria and part of them entered cell nuclei. Moreover, we have demonstrated that both the HA-IR825-Chol-based mitochondrial PTT treatment and the mitochondria-oriented HCPT could destroy mitochondria and trigger cytochrome $\mathrm{c}$ release, and the HCPT in the nuclei could promote cleaved caspase- 3 upregulation, both of which contributed to the cell death. The in vitro and in vivo experiments both proved the synergistic performance of the chemo-photothermal combination therapy. This work exemplifies the development of an intrinsically mitochondria-targetable nanocarrier for precise subcellular structure-localized drug delivery and may deepen the understanding of the relationship between apoptosis and cancer therapy.

\section{Received 8 November 2019; accepted 27 January 2020;} published online 5 March 2020

1 Ott M, Gogvadze V, Orrenius S, et al. Mitochondria, oxidative stress and cell death. Apoptosis, 2007, 12: 913-922 
2 Weinberg SE, Chandel NS. Targeting mitochondria metabolism for cancer therapy. Nat Chem Biol, 2015, 11: 9-15

3 Fulda S, Galluzzi L, Kroemer G. Targeting mitochondria for cancer therapy. Nat Rev Drug Discov, 2010, 9: 447-464

4 Kwon HJ, Cha MY, Kim D, et al. Mitochondria-targeting ceria nanoparticles as antioxidants for alzheimer's disease. ACS Nano, 2016, 10: 2860-2870

5 Lin F, Bao YW, Wu FG. Improving the phototherapeutic efficiencies of molecular and nanoscale materials by targeting mitochondria. Molecules, 2018, 23: 3016

6 Zhou L, Wu Y, Meng X, et al. Dye-anchored MnO nanoparticles targeting tumor and inducing enhanced phototherapy effect via mitochondria-mediated pathway. Small, 2018, 14: 1801008

7 Wang XH, Peng HS, Yang W, et al. Mitochondria-targeted theranostic nanoparticles for optical sensing of oxygen, photodynamic cancer therapy, and assessment of therapeutic efficacy. Microchim Acta, 2016, 183: 2723-2731

8 Tu Z, Qiao H, Yan Y, et al. Directed graphene-based nanoplatforms for hyperthermia: overcoming multiple drug resistance. Angew Chem Int Ed, 2018, 57: 11198-11202

9 Sibrian-Vazquez M, Nesterova IV, Jensen TJ, et al. Mitochondria targeting by guanidine- ${ }^{-}$and biguanidine-porphyrin photosensitizers. Bioconjugate Chem, 2008, 19: 705-713

10 Han K, Lei Q, Wang SB, et al. Dual-stage-light-guided tumor inhibition by mitochondria-targeted photodynamic therapy. Adv Funct Mater, 2015, 25: 2961-2971

11 Ju E, Li Z, Liu Z, et al. Near-infrared light-triggered drug-delivery vehicle for mitochondria-targeted chemo-photothermal therapy. ACS Appl Mater Interfaces, 2014, 6: 4364-4370

12 Zhou F, Wu S, Wu B, et al. Mitochondria-targeting single-walled carbon nanotubes for cancer photothermal therapy. Small, 2011, 7: 2727-2735

13 Liang C, Xu L, Song G, et al. Emerging nanomedicine approaches fighting tumor metastasis: animal models, metastasis-targeted drug delivery, phototherapy, and immunotherapy. Chem Soc Rev, 2016, 45: 6250-6269

14 Mou J, Chen Y, Ma M, et al. Facile synthesis of liposome $/ \mathrm{Cu}_{2-x} \mathrm{~S}-$ based nanocomposite for multimodal imaging and photothermal therapy. Sci China Mater, 2015, 58: 294-301

15 Xing R, Liu K, Jiao T, et al. An injectable self-assembling collagengold hybrid hydrogel for combinatorial antitumor photothermal/ photodynamic therapy. Adv Mater, 2016, 28: 3669-3676

16 Lu X, Li Y, Bai X, et al. Multifunctional $\mathrm{Cu}_{1.94} \mathrm{~S}-\mathrm{Bi}_{2} \mathrm{~S}_{3} @$ polymer nanocomposites for computed tomography imaging guided photothermal ablation. Sci China Mater, 2017, 60: 777-788

17 Gao G, Jiang YW, Jia HR, et al. From perinuclear to intranuclear localization: a cell-penetrating peptide modification strategy to modulate cancer cell migration under mild laser irradiation and improve photothermal therapeutic performance. Biomaterials, 2019, 223: 119443

18 Abadeer NS, Murphy CJ. Recent progress in cancer thermal therapy using gold nanoparticles. J Phys Chem C, 2016, 120: 46914716

19 Liu Z, Cai W, He L, et al. In vivo biodistribution and highly efficient tumour targeting of carbon nanotubes in mice. Nat Nanotech, 2007, 2: 47-52

20 Sun W, Zhang X, Jia HR, et al. Water-dispersible candle sootderived carbon nano-onion clusters for imaging-guided photothermal cancer therapy. Small, 2019, 15: 1804575

21 Kim JW, Galanzha EI, Shashkov EV, et al. Golden carbon nano- tubes as multimodal photoacoustic and photothermal high-contrast molecular agents. Nat Nanotech, 2009, 4: 688-694

22 Jiang BP, Zhou B, Lin Z, et al. Recent advances in carbon nanomaterials for cancer phototherapy. Chem Eur J, 2019, 25: $3993-$ 4004

23 Zhou Z, Yan Y, Wang L, et al. Melanin-like nanoparticles decorated with an autophagy-inducing peptide for efficient targeted photothermal therapy. Biomaterials, 2019, 203: 63-72

24 Mrówczyński R. Polydopamine-based multifunctional (nano)materials for cancer therapy. ACS Appl Mater Interfaces, 2018, 10: 7541-7561

25 Zhang X, Xia LY, Chen X, et al. Hydrogel-based phototherapy for fighting cancer and bacterial infection. Sci China Mater, 2017, 60: 487-503

26 Chen $\mathrm{H}$, Zhang J, Chang K, et al. Highly absorbing multispectral near-infrared polymer nanoparticles from one conjugated backbone for photoacoustic imaging and photothermal therapy. Biomaterials, 2017, 144: 42-52

27 Pierini F, Nakielski P, Urbanek O, et al. Polymer-based nanomaterials for photothermal therapy: from light-responsive to multifunctional nanoplatforms for synergistically combined technologies. Biomacromolecules, 2018, 19: 4147-4167

28 Shao J, Ruan C, Xie H, et al. Black-phosphorus-incorporated hydrogel as a sprayable and biodegradable photothermal platform for postsurgical treatment of cancer. Adv Sci, 2018, 5: 1700848

29 Chen W, Ouyang J, Liu H, et al. Black phosphorus nanosheetbased drug delivery system for synergistic photodynamic/photothermal/chemotherapy of cancer. Adv Mater, 2017, 29: 1603864

30 Wu MX, Yang YW. Metal-organic framework (MOF)-based drug/ cargo delivery and cancer therapy. Adv Mater, 2017, 29: 1606134

31 Liu Y, Zhang Y, Li X, et al. Fluorescence-enhanced covalent organic framework nanosystem for tumor imaging and photothermal therapy. Nanoscale, 2019, 11: 10429-10438

32 Jung HS, Verwilst P, Sharma A, et al. Organic molecule-based photothermal agents: an expanding photothermal therapy universe. Chem Soc Rev, 2018, 47: 2280-2297

33 Yoon HJ, Lee HS, Lim JY, et al. Liposomal indocyanine green for enhanced photothermal therapy. ACS Appl Mater Interfaces, 2017, 9: 5683-5691

34 Chen J, Li X, Liu X, et al. Hybrid $\mathrm{MoSe}_{2}$-indocyanine green nanosheets as a highly efficient phototheranostic agent for photoacoustic imaging guided photothermal cancer therapy. Biomater Sci, 2018, 6: 1503-1516

35 Zhu YX, Jia HR, Gao G, et al. Mitochondria-acting nanomicelles for destruction of cancer cells via excessive mitophagy/autophagydriven lethal energy depletion and phototherapy. Biomaterials, 2020, 232: 119668

36 Chen Z, Zhao P, Luo Z, et al. Cancer cell membrane-biomimetic nanoparticles for homologous-targeting dual-modal imaging and photothermal therapy. ACS Nano, 2016, 10: 10049-10057

37 Zheng M, Yue C, Ma Y, et al. Single-step assembly of DOX/ICG loaded lipid-polymer nanoparticles for highly effective chemophotothermal combination therapy. ACS Nano, 2013, 7: 20562067

38 Jia HR, Zhu YX, Liu X, et al. Construction of dually responsive nanotransformers with nanosphere-nanofiber-nanosphere transition for overcoming the size paradox of anticancer nanodrugs. ACS Nano, 2019, 13: 11781-11792

39 Cheng L, He W, Gong H, et al. PEGylated micelle nanoparticles encapsulating a non-fluorescent near-infrared organic dye as a safe 
and highly-effective photothermal agent for in vivo cancer therapy. Adv Funct Mater, 2013, 23: 5893-5902

40 Yue C, Liu P, Zheng M, et al. IR-780 dye loaded tumor targeting theranostic nanoparticles for NIR imaging and photothermal therapy. Biomaterials, 2013, 34: 6853-6861

41 Luo S, Tan X, Fang S, et al. Mitochondria-targeted small-molecule fluorophores for dual modal cancer phototherapy. Adv Funct Mater, 2016, 26: 2826-2835

42 Zhang J, Liu Z, Lian P, et al. Selective imaging and cancer cell death via $\mathrm{pH}$ switchable near-infrared fluorescence and photothermal effects. Chem Sci, 2016, 7: 5995-6005

43 Camerin M, Rello-Varona S, Villanueva A, et al. Metallo-naphthalocyanines as photothermal sensitisers for experimental tumours: in vitro and in vivo studies. Lasers Surg Med, 2009, 41: 665673

44 Mathew S, Murakami T, Nakatsuji H, et al. Exclusive photothermal heat generation by a gadolinium bis(naphthalocyanine) complex and inclusion into modified high-density lipoprotein nanocarriers for therapeutic applications. ACS Nano, 2013, 7: 8908-8916

45 Lim CK, Shin J, Lee YD, et al. Phthalocyanine-aggregated polymeric nanoparticles as tumor-homing near-infrared absorbers for photothermal therapy of cancer. Theranostics, 2012, 2: 871-879

46 Wei JP, Chen XL, Wang XY, et al. Polyethylene glycol phospholipids encapsulated silicon 2,3-naphthalocyanine dihydroxide nanoparticles ( $\mathrm{SiNcOH}-\mathrm{DSPE}-\mathrm{PEG}\left(\mathrm{NH}_{2}\right) \mathrm{NPs}$ ) for single NIR laser induced cancer combination therapy. Chin Chem Lett, 2017, 28: 1290-1299

47 Jung HS, Lee JH, Kim K, et al. A mitochondria-targeted cryptocyanine-based photothermogenic photosensitizer. J Am Chem Soc, 2017, 139: 9972-9978

48 Zhou B, Li Y, Niu G, et al. Near-infrared organic dye-based nanoagent for the photothermal therapy of cancer. ACS Appl Mater Interfaces, 2016, 8: 29899-29905

49 Pan GY, Jia HR, Zhu YX, et al. Dual channel activatable cyanine dye for mitochondrial imaging and mitochondria-targeted cancer theranostics. ACS Biomater Sci Eng, 2017, 3: 3596-3606

50 Pan GY, Jia HR, Zhu YX, et al. Cyanine-containing polymeric nanoparticles with imaging/therapy-switchable capability for mitochondria-targeted cancer theranostics. ACS Appl Nano Mater, 2018, 1: 2885-2897

51 Jung HS, Han J, Lee JH, et al. Enhanced NIR radiation-triggered hyperthermia by mitochondrial targeting. J Am Chem Soc, 2015, 137: 3017-3023

52 Samali A, Robertson JD, Peterson E, et al. Hsp27 protects mitochondria of thermotolerant cells against apoptotic stimuli. Cell Stress Chaper, 2001, 6: 49-58

53 Gao P, Pan W, Li N, et al. Boosting cancer therapy with organelletargeted nanomaterials. ACS Appl Mater Interfaces, 2019, 11: 26529-26558

54 Kim K, Choi H, Choi ES, et al. Hyaluronic acid-coated nanomedicine for targeted cancer therapy. Pharmaceutics, 2019, 11: 301

55 Lapčík Jr L, Lapćík L, De Smedt S, et al. Hyaluronan: preparation, structure, properties, and applications. Chem Rev, 1998, 30: 26632684

56 Choi KY, Chung H, Min KH, et al. Self-assembled hyaluronic acid nanoparticles for active tumor targeting. Biomaterials, 2010, 31: 106-114

57 Song E, Han W, Li C, et al. Hyaluronic acid-decorated graphene oxide nanohybrids as nanocarriers for targeted and $\mathrm{pH}$-responsive anticancer drug delivery. ACS Appl Mater Interfaces, 2014, 6:
11882-11890

58 Zhang L, Gao S, Zhang F, et al. Activatable hyaluronic acid nanoparticle as a theranostic agent for optical/photoacoustic imageguided photothermal therapy. ACS Nano, 2014, 8: 12250-12258

59 Gao S, Wang J, Tian R, et al. Construction and evaluation of a targeted hyaluronic acid nanoparticle/photosensitizer complex for cancer photodynamic therapy. ACS Appl Mater Interfaces, 2017, 9: 32509-32519

60 Hyung W, Ko H, Park J, et al. Novel hyaluronic acid (HA) coated drug carriers (HCDCs) for human breast cancer treatment. Biotechnol Bioeng, 2008, 99: 442-454

61 Lim EK, Kim HO, Jang E, et al. Hyaluronan-modified magnetic nanoclusters for detection of CD44-overexpressing breast cancer by MR imaging. Biomaterials, 2011, 32: 7941-7950

62 Choi KY, Min KH, Yoon HY, et al. PEGylation of hyaluronic acid nanoparticles improves tumor targetability in vivo. Biomaterials, 2011, 32: 1880-1889

63 Jia HR, Zhu YX, Xu KF, et al. Plasma membrane-anchorable photosensitizing nanomicelles for lipid raft-responsive and lightcontrollable intracellular drug delivery. J Control Release, 2018, 286: 103-113

64 Jia HR, Zhu YX, Xu KF, et al. Efficient cell surface labelling of live zebrafish embryos: wash-free fluorescence imaging for cellular dynamics tracking and nanotoxicity evaluation. Chem Sci, 2019, 10: $4062-4068$

65 Wei X, Senanayake TH, Warren G, et al. Hyaluronic acid-based nanogel-drug conjugates with enhanced anticancer activity designed for the targeting of CD44-positive and drug-resistant tumors. Bioconjugate Chem, 2013, 24: 658-668

66 Seo YJ, Jeong HS, Bang EK, et al. Cholesterol-linked fluorescent molecular beacons with enhanced cell permeability. Bioconjugate Chem, 2006, 17: 1151-1155

67 Whitehouse WL, Noble JE, Ryadnov MG, et al. Cholesterol anchors enable efficient binding and intracellular uptake of DNA nanostructures. Bioconjugate Chem, 2019, 30: 1836-1844

68 Pan GY, Jia HR, Zhu YX, et al. Turning double hydrophilic into amphiphilic: IR825-conjugated polymeric nanomicelles for nearinfrared fluorescence imaging-guided photothermal cancer therapy. Nanoscale, 2018, 10: 2115-2127

69 Gao G, Jiang YW, Sun W, et al. Molecular targeting-mediated mild-temperature photothermal therapy with a smart albuminbased nanodrug. Small, 2019, 15: 1900501

70 Garcia-Carbonero R, Supko JG. Current perspectives on the clinical experience, pharmacology, and continued development of the camptothecins. Clin Cancer Res, 2002, 8: 641-661

71 Yang L, Cui F, Cun D, et al. Preparation, characterization and biodistribution of the lactone form of 10-hydroxycamptothecin (HCPT)-loaded bovine serum albumin (BSA) nanoparticles. Int J Pharm, 2007, 340: 163-172

72 Sánchez-Alcázar JA, Ault JG, Khodjakov A, et al. Increased mitochondrial cytochrome $\mathrm{c}$ levels and mitochondrial hyperpolarization precede camptothecin-induced apoptosis in jurkat cells. Cell Death Differ, 2000, 7: 1090-1100

73 Fulda S. Apoptosis pathways and neuroblastoma therapy. Curr Pharm Design, 2009, 15: 430-435

74 Vakifahmetoglu-Norberg H, Ouchida AT, Norberg E. The role of mitochondria in metabolism and cell death. Biochem BioPhys Res Commun, 2017, 482: 426-431

75 Bank A, Wang P, Du C, et al. SMAC mimetics sensitize nonsteroidal anti-inflammatory drug induced apoptosis by promoting 
caspase-3 mediated cytochrome c release. Cancer Res, 2008, 68: 276-284

76 Ow YLP, Green DR, Hao Z, et al. Cytochrome c: functions beyond respiration. Nat Rev Mol Cell Biol, 2008, 9: 532-542

77 Porter AG, Jänicke RU. Emerging roles of caspase-3 in apoptosis. Cell Death Differ, 1999, 6: 99-104

78 Tang W, Zhang Y, Qian C, et al. Induction and mechanism of apoptosis by hydroxycamptothecin in human tenon's capsule fibroblasts. Invest Ophthalmol Vis Sci, 2012, 53: 4874-4880

Acknowledgements This work was supported by the National Natural Science Foundation of China (21673037).

Author contributions Liu X, Zhu Y, Xu K, and Yu X performed the experiments. Liu X, Jia H, Gao G, Jiang Y, and Wu F analyzed the data. Liu X, Jia H, Zhu Y, Cheng X, and Wu F wrote the manuscript. Wu F supervised the project.

Conflict of interest The authors declare that they have no conflict of interest.

Supplementary information Experimental details and supporting data are available in the online version of the paper.

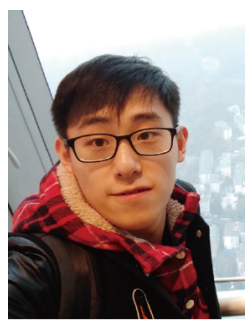

Xiaoyang Liu received his BSc degree from Northeastern University in 2017. In 2015, he became a visiting student at Shenzhen Institute of Advanced Technology, Chinese Academy of Sciences, and carried out research on photoacoustic imaging. He is currently a postgraduate student at Southeast University under the supervision of Prof. Fu-Gen Wu and Prof. Zhan Chen. His current research is focused on the nanomedicine for cancer therapy.

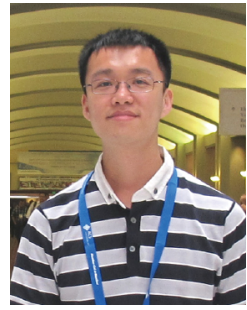

Fu-Gen $\mathbf{W u}$ is a professor of biomedical en gineering at Southeast University, Nanjing, China. He obtained his $\mathrm{BSc}$ and $\mathrm{PhD}$ degrees from Tsinghua University in 2006 and 2011, respectively. After a postdoctoral period at the University of Michigan-Ann Arbor, he joined Southeast University in 2013 and was promoted to be a professor. His main research interests are biomaterials, nanomedicine, and cell imaging.

\section{作用于线粒体和细胞核的聚合物纳米材料及其在 化疗/光热治疗联合抗癌中的应用}

刘篗阳, 贾浩然, 祝雅璇, 高歌, 蒋耀文, 程晓㣌, 许可飞, 于心望, 吴富根

摘要 开发本身即具有线粒体靶向能力的亚细胞精准纳米诊疗试 剂对于改善癌症治疗效果具有重要意义. 本文使用可靶向癌细胞 表面过度表达的 CD44抗原的透明质酸、胆固醇-聚乙二醇-氨基和 可作用于线粒体的花菁类染料IR825- $\mathrm{NH}_{2}$, 构建了一种可实现光热 治疗的自组装纳米材料(HA-IR825-Chol). 相较于游离的IR825$\mathrm{NH}_{2}$, 该结构具有更好的光稳定性、更高的光热转换效率和对癌细 胞的识别能力. HA-IR825-Chol可以有效靶向细胞线粒体, 并可以 在近红外激光照射下诱导线粒体损伤. 此外, 我们通过疏水作用包 裹了化疗试剂 10 - 差基喜树碱 (HCPT)(所形成的药物命名为 HAIR825-Chol/HCPT). 相关实验结果显示, 包裏于纳米材料后 HCPT 被细胞摄取的效率显著提高, 并能够同时分布于线粒体和细胞核 中, 从而诱导线粒体中细胞色素 c的释放和细胞中cleaved caspase-3 的上调, 最终促进细胞调亡与死亡. 另外, HA-IR825-Chol/HCPT优 异的体内肿瘤靶向能力为光化疗联合治疗消除肿瘤提供了必要保 证. 该工作实现了定位于线粒体的精准亚细胞药物递送, 并发展了 利用胆固醇提高药物摄取速率和效率的策略, 预期将为提高纳米 药物抗癌效果提供借鉴. 